\title{
Estimating the population health burden of Lyme disease in Ontario, Canada: a microsimulation modelling approach
}

\author{
Stephen Mac MBiotech, Gerald A. Evans MD, Samir N. Patel PhD, Eleanor M. Pullenayegum PhD, \\ Beate Sander $\mathrm{PhD}$
}

\section{Abstract}

Background: If untreated, Lyme disease can lead to long-term sequelae and post-treatment Lyme disease syndrome (PTLDS), resulting in reduced health-related quality of life. The objective of this study was to develop a microsimulation model to estimate the population-level health burden of Lyme disease in Ontario, Canada.

\begin{abstract}
Methods: We developed a Lyme disease history model using microsimulation, simulating 100000 people (mean age 37.6 yr, $51 \%$ female) from 2017 in Ontario over a lifetime risk of infection and time horizon. We extracted the sensitivity and specificity of the 2-tier testing recommended by the Canadian Public Health Laboratory Network, probabilities and health state utility values from the published literature and health administrative data. Our reported outcomes from our stochastic analysis include diagnosed cases of Lyme disease (stratified by stage), undiagnosed infections, sequelae, individuals with PTLDS and quality-adjusted life-years (QALYs) lost.

Results: Our model estimated 333 (95\% confidence interval [Cl] 329-337) infections over the lifetime of 100000 simulated people (mean age $37.6 \mathrm{yr}, 51 \%$ female), with $92 \%(95 \% \mathrm{Cl} 91 \%-93 \%)$ of infections diagnosed. Of those 308 people with Lyme Disease diagnoses, 67 (95\% Cl 65-69) developed sequelae (e.g., arthritic, cardiac, neurologic sequelae), and 34 (95\% Cl 33-35) developed PTLDS. Lyme disease resulted in a loss of 84.5 QALYs (95\% CI 82.9-86.2) over the lifetime of the simulated cohort. Sensitivity and scenario analysis showed that increasing incidence rates of Lyme disease, potential underreporting, duration of PTLDS and quality of life (health state utility) associated with PTLDS had the greatest impact on health burden.
\end{abstract}

Interpretation: Lyme disease contributes considerable health burden in terms of QALYs lost. Our analysis provides evidence to understand the disease burden and lays the foundation to assess the cost-effectiveness of pharmaceutical and nonpharmaceutical interventions.

\section{L} yme disease is the most commonly reported vectorborne disease in North America, ${ }^{1-3}$ with annual incidence rates of up to 130 per 100000 people in Maine, United States, ${ }^{4}$ and 85 per 100000 people in highrisk areas of Ontario, Canada. ${ }^{5}$ If Lyme disease is untreated in its early stages, it can lead to Lyme meningitis, cranial nerve neuropathies (e.g., Bell palsy), Lyme carditis and Lyme arthritis. ${ }^{6}$ Treated patients may be at risk of posttreatment Lyme disease syndrome (PTLDS), defined as persistent symptoms for at least 6 months after treatment of Lyme disease that was documented by a physician and treated with standard-of-care antibiotics. ${ }^{7}$ A systematic review of long-term sequelae and health-related quality of life of patients with confirmed Lyme disease showed that PTLDS may result in impaired quality of life. ${ }^{8}$

Three Markov cohort models have been developed to assess the cost-effectiveness of vaccination for Lyme disease, ${ }^{9-11}$ and a fourth model was developed to assess the costeffectiveness of oral antibiotic therapy. ${ }^{12}$ However, these models assumed a homogeneous population, did not incorporate the issue of Lyme disease diagnosis (e.g., clinical v. diagnostic tests, stage of Lyme disease and associated test sensitivity), individual-level characteristics or the entire disease progression of Lyme disease (i.e., PTLDS).

The individual- and population-level burdens of Lyme disease are not well understood for several reasons, including the relative novelty of the disease in certain regions of North America, low incidence rates and lack of follow-up on longterm outcomes. In these instances, microsimulation models can simulate the disease history of Lyme disease based on current evidence from the literature and surveillance programs to inform estimates of its impact on health outcomes. New models should include updated case definitions, diagnostic effectiveness, treatment and disease progression. The objective of

\section{Competing interests: None declared.}

This article has been peer reviewed.

Correspondence to: Stephen Mac,sm.mac@mail.utoronto.ca

CMAJ Open 2021 November 16. DOI:10.9778/cmajo.20210024 
this study was to estimate the health burden of Lyme disease, including cases, outcomes and quality-adjusted life-years (QALYs), in Ontario, Canada, using an individual-level, statetransition model.

\section{Methods}

\section{Design, setting and population}

We developed an individual-level, state-transition model to simulate a cohort representative of the population of Ontario in 2017.

We simulated a cross-sectional cohort of 100000 people in Ontario in accordance with Ontario demographics (mean $37.6 \mathrm{yr}$, standard deviation [SD] $22.7 \mathrm{yr}, 51 \%$ female), representing $63 \%$ of the total population (8.8 million of 14 million) residing in high-risk areas (Mark Nelder, Public Health Ontario, Toronto, Ont.: personal communication, 2021). The average life expectancy of Ontarians of our cohort was 81.0 years, simulating a lifetime risk of Lyme disease of about 43 years.

We sampled individual characteristics (i.e., age, sex, risk area of residence) from distributions informed by census data (details about sampling are described in Appendix 1, Section 1, available at www.cmajopen.ca/content/9/4/E1005/suppl/ DC1). ${ }^{13}$ We designated possible health states as healthy, undiagnosed infection, diagnosed Lyme disease at various stages of disease or recovery. The model used weekly cycle lengths (i.e., each timestep in the model represents a week).

\section{Outcomes}

We reported health outcomes per 100000 people, including the number of cases of Lyme disease (diagnosed at the early localized, early disseminated and late disseminated stages), frequency of different types of sequelae (including PTLDS) and QALYs. Outcomes were accrued over the patient's lifetime and discounted at $1.5 \%$ to account for time preference of health outcomes. ${ }^{14} \mathrm{We}$ conducted all modelling and analyses using Treeage.

\section{Model structure}

\section{Lyme disease incidence and exposure}

We assumed that individuals enter the model in a healthy (susceptible) state (Figure 1), with a probability that these individuals can be bit by an infected tick and develop Lyme disease according to age- and sex-dependent incidence rates. Age-dependent incidence rates ranged between 2.9 and 10 per 100000 females, and 5.0 and 13.9 per 100000 males in 2017 (Appendix 1, Section 2). ${ }^{5}$

People who develop an erythema migrans rash may receive a clinical diagnosis and transition to the early localized health state. Those who present with erythema migrans can be misdiagnosed depending on clinician awareness of Lyme disease, ${ }^{13}$ and remain undiagnosed in the undiagnosed infection state, where they may have decreased quality of life, eventually receive a diagnosis or remain undiagnosed. The likelihood of

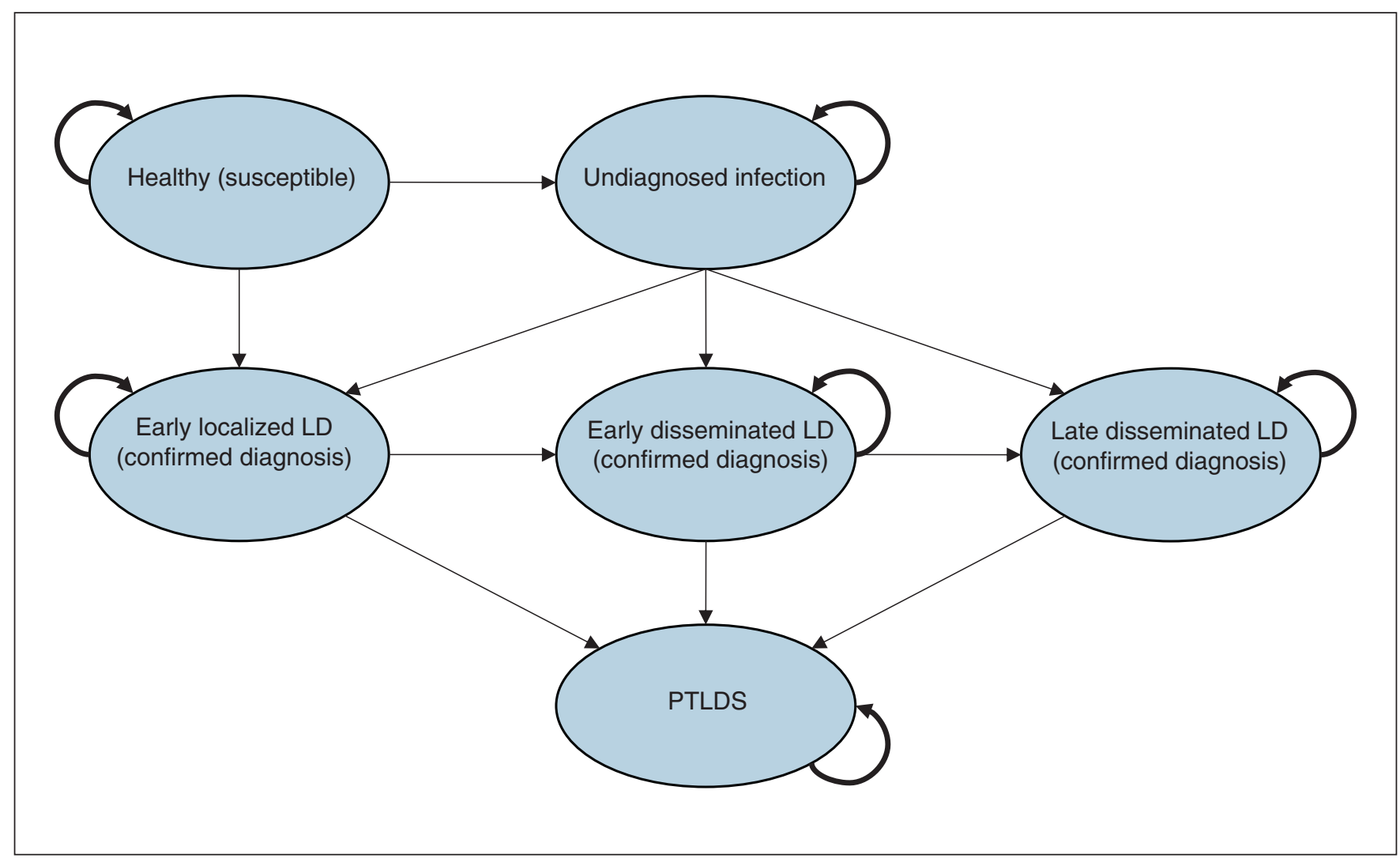

Figure 1: Model schematic of individual state-transition model. Note: LD = Lyme disease, PTLDS = post-treatment Lyme disease syndrome. Within the states of Lyme disease, individuals can develop sequelae. Individuals can recover to a healthy state from any state of Lyme disease. 
clinical diagnosis after presenting with erythema migrans in high- and low-exposure areas was $58 \%$ and $26 \%$, respectively. ${ }^{15}$ Once a diagnostic test confirms Lyme disease, individuals transition to the early localized, early disseminated or late disseminated health states, depending on the time since initial infection, which is defined in this model as between 0 and 30 days, 31 and 90 days, and 91 days and onwards, respectively. ${ }^{16}$

\section{Diagnostic testing}

The model incorporated 2-tier tests (e.g., immunoglobulin [Ig] M/IgG ELISA, Western blot), recommended by the Canadian Public Health Laboratory Network. ${ }^{17}$ Two-tier tests with negative or positive results are returned within 1 or 2 weeks, respectively. ${ }^{17}$ Individuals in the undiagnosed infection state have a weekly probability of getting tested depending on whether or not they develop sequelae. An average of $80.4 \%$ ( $63 \%$ to $98 \%$, depending on sequelae) of individuals would be tested if presenting with sequelae from the initial infection, and $40.2 \%$ if not. ${ }^{15}$

We extracted sensitivity and specificity from a metaanalysis, stratified by stage of Lyme disease. The sensitivity for early localized, early disseminated and late disseminated Lyme disease was $46.3 \%, 89.7 \%$ and $99.4 \%$, respectively, and the specificity was between $99.3 \%$ and $99.7 \% .^{18}$

\section{Sequelae and manifestations of Lyme disease}

In the early disseminated stage, multiple erythema migrans, cardiac abnormalities, cranial nerve palsies and other neurologic sequelae (meningitis and polyneuropathy) can develop, whereas in the late disseminated stage, arthritic and cognitive sequelae can develop. ${ }^{8}$ The 1-year, sex-dependent probability of developing early disseminated and late disseminated stage sequelae was $17 \%$, and $10 \%$ to $11 \%$, respectively, as informed by population-based health administrative data in Ontario (Table 1, details in Appendix 1, Section 3). Hospitalizations from disseminated stages of Lyme disease can result in lower quality of life for a short period.

Individuals with clinically diagnosed Lyme disease or with laboratory confirmation receive 2 to 3 weeks of oral antibiotics, which may be followed by subsequent courses of intravenous antibiotics, depending on sequelae. ${ }^{27}$ We define treatment success as an absence of sequelae or manifestations of the respective stage of Lyme disease (i.e., no more persistent symptoms). On treatment, individuals have a $4 \%$ to $6 \%$ chance of having minor or major adverse events from antibiotic treatment, ${ }^{12}$ and an $85 \%$ to $95 \%$ chance of recovery. ${ }^{19-24}$ Unsuccessful treatment leads to possible development of PTLDS; we assumed, with expert guidance, that PTLDS persists for 5 years. After recovery from any stage of Lyme disease, individuals are assumed to be immune from reinfection for 6 months.

\section{Utilities}

Health state utility values are preference values for being in a health state, used to measure the quality of life (morbidity) in conjunction with life-years to output QALYs. ${ }^{28}$ Mean utility values for the Ontario population ranged from 0.62 to 0.90 , depending on age and sex. ${ }^{13} \mathrm{We}$ extracted utilities for the health states of Lyme disease from a systematic review, ${ }^{8}$ corresponding to the experienced sequelae. For instance, an individual with cranial nerve palsy would have a utility of 0.61 , whereas PTLDS is associated with a utility of $0.54 .{ }^{10}$ All parameters are summarized in Table 1.

\section{Statistical analysis}

We ran 100 simulations of 100000 individuals with a risk of Lyme disease infection throughout their lifetime, assuming that treatment starts after diagnosis (clinical or laboratory diagnosis). We calculated the mean (95\% confidence intervals $[\mathrm{CIs}]$ ) and median (interquartile range [IQR]) incidence for outcomes per 100000 people.

\section{Assessing uncertainty}

For scenario and sensitivity analyses, we used a seeded deterministic simulation for computational efficiency and to illustrate the impact of the variable of interest, within its specified range, on the results, while keeping all other parameters and processes identical (Table 1). In the scenario of increasing incidence rates of Lyme disease, we increased the incidence annually by 1 per 100000 people for each age group for the subsequent 10 years. All scenarios are described in Appendix 1, Section 4.

We also simulated the health burden for 1 million people (using a similar mean age, sex and proportion of endemic residence in Ontario in 2017), over a 1-year risk of Lyme disease to contextualize annual health burden. We conducted a deterministic 1-way sensitivity analysis to assess the robustness of model results to key parameters and associated uncertainty. We followed modelling best practices; 2 clinical experts (authors G.A.E. and S.N.P.) internally validated the model, which we also externally validated to observational data from the literature. ${ }^{29}$

\section{Ethics approval}

We did not require ethics approval for this type of modelling study.

\section{Results}

Using our cross-sectional cohort of 100000 people in Ontario, we estimated a mean of 333 (95\% CI 329-337) infections per 100000 people, of which 4 (95\% CI 3-4; 1.3\%) were reinfections. From these 333 people with infections, an average of 308 (95\% CI 304-311; 92\%) had Lyme disease diagnosed, and 26 (95\% CI 25-27; 8\%) remained undiagnosed. All results are summarized in Table 2.

The mean age at the time of infection was 54.6 (SD 17.9) years, with most infections in the 50-55 and 60-65 year age groups (Appendix 1, Section 5). The median duration of Lyme disease infection was 7 (range 2-260) weeks (Appendix 1, Section 5). The median time from infection to diagnosis, and from infection to treatment was 5 (IQR 3-6), and 6 (IQR 4-7) weeks, respectively (Appendix 1, Section 5). 
Research

Of the people with diagnoses, an average of 107 (95\% CI $105-109 ; 32 \%)$ were diagnosed at the early localized stage, of which 44 (95\% CI 43-46; 13\%) were clinically diagnosed, and 63 (95\% CI 62-64; 19\%) were diagnosed through laboratory confirmation. Laboratory-confirmed diagnosis of early disseminated and late disseminated cases occurred in an average of 137 (95\% CI 134-140; 41\%), and 63 (95\% CI 61-65; 19\%) infections, respectively.

\section{Table 1 (part 1 of 2): Key parameters and data sources}

\begin{tabular}{|c|c|c|}
\hline Parameter & Base case value (range) ${ }^{*}$ & Data sources \\
\hline \multicolumn{3}{|c|}{ Key Lyme disease probability parameters and data sources } \\
\hline \multicolumn{3}{|l|}{ Lyme disease } \\
\hline Probability of high-risk exposure & $0.628(0.471-0.786) \dagger$ & Personal communication, $\mathrm{PHO}$ \\
\hline $\begin{array}{l}\text { LD incidence rates, per } 100000 \text { (varies by age } \\
\text { and sex) }\end{array}$ & $2.9-13.9(0.000029-0.000139) \ddagger$ & Nelder et al. $2018^{5}$ \\
\hline $\begin{array}{l}\text { Probability of clinical diagnosis after EM rash, } \\
\text { high-risk exposure area }\end{array}$ & $0.583(0.437-0.729) \ddagger$ & Henry et al. $2012^{15}$ \\
\hline $\begin{array}{l}\text { Probability of clinical diagnosis after } \\
\text { EM rash, low-risk exposure area }\end{array}$ & $0.261(0.196-0.326) \dagger$ & Henry et al. $2012^{15}$ \\
\hline \multicolumn{3}{|l|}{ Diagnostics } \\
\hline Sensitivity, early localized & $0.463(0.391-0.537) \S$ & Waddell et al. $2016^{18}$ \\
\hline Sensitivity, early disseminated & $0.897(0.783-0.954) \S$ & Waddell et al. $2016^{18}$ \\
\hline Sensitivity, late disseminated & $0.994(0.957-0.999) \S$ & Waddell et al. $2016^{18}$ \\
\hline Specificity, early localized & $0.993(0.983-0.997) \S$ & Waddell et al. $2016^{18}$ \\
\hline Specificity, early disseminated & $0.997(0.984-0.999) \S$ & Waddell et al. $2016^{18}$ \\
\hline Specificity, late disseminated & $0.993(0.985-0.997) \S$ & Waddell et al. $2016^{18}$ \\
\hline $\begin{array}{l}\text { Probability of testing (varies by presence or } \\
\text { absence of sequelae) }\end{array}$ & $0.402-0.805(0.30-0.98) \dagger$ & Henry et al. $2012^{15}$ \\
\hline Delay in results & $1-2 \mathrm{wk}$ & PHO $2017^{17}$ \\
\hline \multicolumn{3}{|l|}{ Treatment } \\
\hline \multicolumn{3}{|l|}{ Treatment efficacy } \\
\hline Erythema migrans & $0.85(0.80-1.00) \ddagger$ & Magid et al. $1992^{19}$ \\
\hline Arthritic sequelae & $0.85(0.40-0.80) \ddagger$ & Liu et al. $1989^{20}$ \\
\hline Cardiac sequelae & $0.90(0.80-1.00) \ddagger$ & Steere et al. $1993^{21}$ \\
\hline Neurologic sequelae & $0.90(0.76-0.97) \ddagger$ & $\begin{array}{c}\text { Logigian and Steere } 1992^{22} \text { Dattwyler et al. } 1988^{23} \\
\text { Karlsson et al. } 1994^{24}\end{array}$ \\
\hline Oral treatment completion & $0.90(0.68-1.00) \dagger$ & Magid et al. $1992^{19}$ \\
\hline IV treatment completion & $0.99(0.75-1.00) \dagger$ & Magid et al. $1992^{19}$ \\
\hline Probability of adverse event, oral & $0.04(0.03-0.05) \dagger$ & Shadick et al. $2001^{10}$ \\
\hline Probability of adverse event, IV & $0.06(0.05-0.08) \dagger$ & Shadick et al. $2001^{10}$ \\
\hline \multicolumn{3}{|l|}{ Outcomes } \\
\hline Probability of hospitalization & $0.05(0.04-0.06) \dagger$ & Shing et al. $2019^{25}$ \\
\hline Length of hospitalization, $d$ & $7.9(3.8-12.1) \S$ & Shing et al. $2019^{25}$ \\
\hline EM rash & $0.80(0.60-1.00) \dagger$ & Shadick et al. $2001^{10}$ \\
\hline $\begin{array}{l}\text { Probability of developing sequelae (varies by } \\
\text { LD stage and sex) }\end{array}$ & $0.10-0.17(0.08-0.21) \dagger$ & Unpublished data from cited study ${ }^{26 * *}$ \\
\hline Arthritic sequelae (M, F) & $0.56-0.63(0.41-0.76) \dagger$ & Unpublished data from cited study ${ }^{26 * *}$ \\
\hline Cardiac sequelae (F, M) & $0.43-0.48(0.29-0.53) \dagger$ & Unpublished data from cited study ${ }^{26 * *}$ \\
\hline Cognitive sequelae $(\mathrm{F}, \mathrm{M})$ & $0.37-0.44(0.29-0.58) \dagger$ & Unpublished data from cited study ${ }^{26 * \star}$ \\
\hline Cranial nerve palsy sequelae $(F, M)$ & $0.11-0.24(0.08-0.26) \dagger$ & Unpublished data from cited study ${ }^{26 * *}$ \\
\hline Multiple EM sequelae (M, F) & $0.22-0.36(0.16-0.40) \dagger$ & Unpublished data from cited study ${ }^{26 * *}$ \\
\hline Meningitis or polyneuropathy sequelae (F, M) & $0.06-0.11(0.12-0.24) \dagger$ & Unpublished data from cited study ${ }^{26 * *}$ \\
\hline
\end{tabular}




\begin{tabular}{|c|c|c|}
\hline Parameter & Base case value (range) ${ }^{*}$ & Data sources \\
\hline \multicolumn{3}{|c|}{ Key utility parameters and data sources } \\
\hline \multicolumn{3}{|l|}{ Utilities } \\
\hline Healthy, stratified by age and sex & $0.62-0.90(0.38-0.98) \S$ & Guertin et al. $2018^{13}$ \\
\hline Arthritic sequelae & $0.69(0.51-0.86) \emptyset$ & Shadick et al. $2001^{10}$ \\
\hline Cardiac sequelae & $0.61(0.38-0.78) \emptyset$ & Shadick et al. $2001^{10}$ \\
\hline Cognitive sequelae & $0.60(0.37-0.73) \emptyset$ & Shadick et al. $2001^{10}$ \\
\hline Erythema migrans & $0.80(0.70-0.93)$ | & Shadick et al. $2001^{10}$ \\
\hline Cranial nerve palsy & $0.61(0.36-0.81) \emptyset$ & Shadick et al. $2001^{10}$ \\
\hline Meningitis or polyneuropathy & $0.52(0.27-0.73) \emptyset$ & Shadick et al. $2001^{10}$ \\
\hline PTLDS & $0.54(0.30-0.70)$ | & Shadick et al. $2001^{10}$ \\
\hline Minor adverse events, disutility & $0.05(0.04-0.06) \dagger$ & Eckman et al. $1997^{12}$ \\
\hline Major adverse events, disutility & $0.10(0.08-0.13) \dagger$ & Eckman et al. $1997^{12}$ \\
\hline Oral treatment, disutility & $0.01(0.00-0.01) \dagger$ & Eckman et al. $1997^{12}$ \\
\hline Intravenous treatment, disutility & $0.03(0.02-0.04) \dagger$ & Eckman et al. $1997^{12}$ \\
\hline \multicolumn{3}{|c|}{ 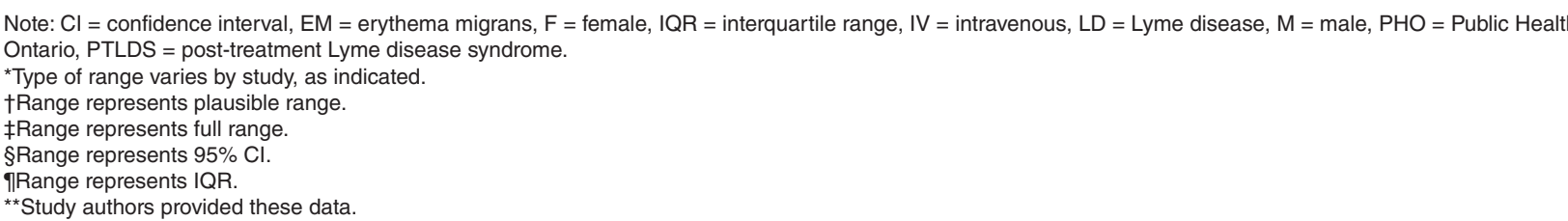 } \\
\hline
\end{tabular}

Within the early disseminated stage, 12 (95\% CI 12-13) people developed disseminated or multiple erythema migrans, 8 (95\% CI 7-8) developed cranial nerve palsy, 20 (95\% CI 19-20) developed cardiac abnormalities and 4 (95\% CI 3-4) developed other neurologic sequelae (e.g., meningitis, polyneuropathy). Of those with late disseminated Lyme disease, 14 (95\% CI 13-15) developed arthritis and 9 (95\% CI 9-10) developed cognitive sequelae. Of all people with diagnoses, 34 (95\% CI 33-35) developed PTLDS. Over the lifetime of 100000 people, Lyme disease resulted in a mean loss of 84.5 (95\% CI 82.9-86.1) QALYs, discounted at $1.5 \%$, or 112.6 (109.6-115.4) QALYs undiscounted.

In the deterministic simulation of the cross-sectional cohort of 1 million people (Appendix, Section 6), there were 53 Lyme disease infections, mostly diagnosed at the early localized (40\%) and early disseminated stages (38\%). About $9 \%$ of people had a diagnosis of PTLDS, and no one was reinfected, which was as predicted, given the low probability of being reinfected within 1 year. In this scenario, 1 year of risk of Lyme disease infection resulted in 19.4 QALYs lost. For Ontario (with its population of about 14 million), this translates to 271 QALYs lost in 1 year.

\section{Assessing uncertainty}

The tornado diagram (Figure 2) summarizes multiple 1-way sensitivity analyses of the impact of all parameters on QALYs lost using a seeded simulation (i.e., 1 constant simulation), where 70.6 QALYs were lost because of Lyme disease. The following parameters (within their plausible range) have the most impact: duration of the PTLDS stage, utility value for PTLDS, probability of full recovery after treatment, probability of developing an erythema migrans rash and diagnostic test performance.

When the duration of PTLDS state was 1 year, 50.4 QALYs are lost, whereas being in the PTLDS health state for an average of 10 years resulted in 87.8 QALYs lost. At the higher estimate for PTLDS utility value of 0.70 , 58.3 QALYs were lost. A lower utility value of 0.30 resulted in 89.1 QALYs lost. With the probability of recovery at its upper limit (e.g., close to $100 \%$ chance of recovery), there is a total of 68.5 QALYs lost, whereas the lower limit resulted in 86.0 QALYs lost.

The other major influential parameter was the incidence of Lyme disease (Appendix 2). In a scenario where incidence rates increased by 1 per 100000 people annually over the subsequent 10 years, 165.5 QALYs were lost. Underreporting factors of 3 (Canadian estimate), and 10 (US estimate) resulted in 257.5 and 881.3 QALYs lost, respectively (Appendix 1, Section 4).

\section{Interpretation}

We developed an individual-level state-transition model to estimate the health burden of Lyme disease over the lifetime of 100000 people in Ontario. Lyme disease resulted in 84 QALYs lost, with $22 \%$ of people developing sequelae and 
Research

\begin{tabular}{|c|c|c|c|c|}
\hline Outcomes & $\begin{array}{l}\text { Mean incidence per } \\
100000(95 \% \mathrm{Cl})\end{array}$ & $\begin{array}{l}\text { Proportion of mean } \\
\text { incidence of total LD } \\
\text { infections, \%* }\end{array}$ & $\begin{array}{l}\text { Median incidence } \\
\text { per } 100000(\mathrm{IQR})\end{array}$ & $\begin{array}{l}\text { Proportion of median } \\
\text { incidence of total LD } \\
\text { infections, \%* }\end{array}$ \\
\hline Total LD infections & 333 (329-337) & & $334(320-346)$ & \\
\hline Diagnosed cases & $308(304-311)$ & 92.5 & $308(294-321)$ & 92.2 \\
\hline Clinically diagnosed & $44(43-46)$ & 13.2 & $45(38-49)$ & 13.5 \\
\hline Laboratory-confirmed & $63(62-64)$ & 18.9 & $63(58-68)$ & 18.9 \\
\hline Early disseminated (laboratory-confirmed) & $137(134-140)$ & 41.1 & $137(129-144)$ & 41.0 \\
\hline Late disseminated (laboratory-confirmed) & $63(61-65)$ & 18.9 & $63(58-69)$ & 18.9 \\
\hline Undiagnosed cases & $26(25-27)$ & 7.8 & $26(22-29)$ & 7.8 \\
\hline Cardiac & $20(19-20)$ & 6.0 & $19(17-22)$ & 5.9 \\
\hline Cranial nerve palsy & $8(7-8)$ & 2.4 & $9(6-10)$ & 2.7 \\
\hline Multiple erythema migrans & $12(12-13)$ & 3.6 & $12(10-15)$ & 3.6 \\
\hline $\begin{array}{l}\text { Neurological (meningitis, } \\
\text { polyneuropathy) }\end{array}$ & $4(3-4)$ & 1.2 & $3(2-5)$ & 0.9 \\
\hline \multicolumn{5}{|l|}{ Late disseminated stage } \\
\hline Arthritic & $14(13-15)$ & 4.2 & $14(12-16)$ & 4.2 \\
\hline Cognitive & $9(9-10)$ & 2.7 & $9(7-11)$ & 2.7 \\
\hline \multicolumn{5}{|l|}{ All stages } \\
\hline
\end{tabular}

10\% developing PTLDS. The duration and quality of life associated with PTLDS had a substantial effect on the overall burden. Our sensitivity analyses suggest that the disease progression toward PTLDS substantially contributes to the health burden associated with Lyme disease infections. Further research and understanding of PTLDS can aid in reducing the health burden of Lyme disease.

Our model was internally validated by clinical experts and was compared with the literature for external validation using health outcomes and estimated QALYs lost. We estimated that $10 \%$ of cases develop PTLDS, which is consistent with the lower range of what has been reported in the literature $(10 \%-20 \%) .{ }^{30}$ Since reinfection rates in Ontario, Canada are not accessibly reported, our $1.3 \%$ estimated probability of reinfection was similar to estimates of 1.2 to $1.7 \%$ for adults in the US, ${ }^{31}$ and lower than $3.4 \%$ in hyperendemic areas in the US. ${ }^{32}$

We also compared our model to a descriptive study of cases of Lyme disease in Ontario from 2005 to 2014. The proportion of people notifying public health systems within 30 days, 1-3 months and > 3 months of symptom onset was $45.2 \%, 38 \%$ and $16.8 \%$, respectively. ${ }^{33}$ Our model similarly estimated that $32 \%$ of diagnosed (and reported) cases were diagnosed within 30 days, $41 \%$ were within $1-3$ months, and $19 \%$ were after 3 months. The slight differences between our results can be attributed to how our model considers the stage of Lyme disease at diagnosis based on time from tick bite or infection, and sequelae developed after infection, which is difficult to identify using administrative data.

Estimates of QALYs lost from Lyme disease and other infectious diseases are limited. An Ontario Burden of Infectious Diseases study from 2012 estimated the annual burden of 51 infectious diseases in health-adjusted life-years (HALYs), a composite measure similar to the QALY that incorporates both mortality (years of life lost) and morbidity (year-equivalents of reduced functioning). ${ }^{34}$ In an indirect comparison, the annual burden of Lyme disease (271 QALYs) is similar in magnitude to herpes simplex virus (256 HALYs) 


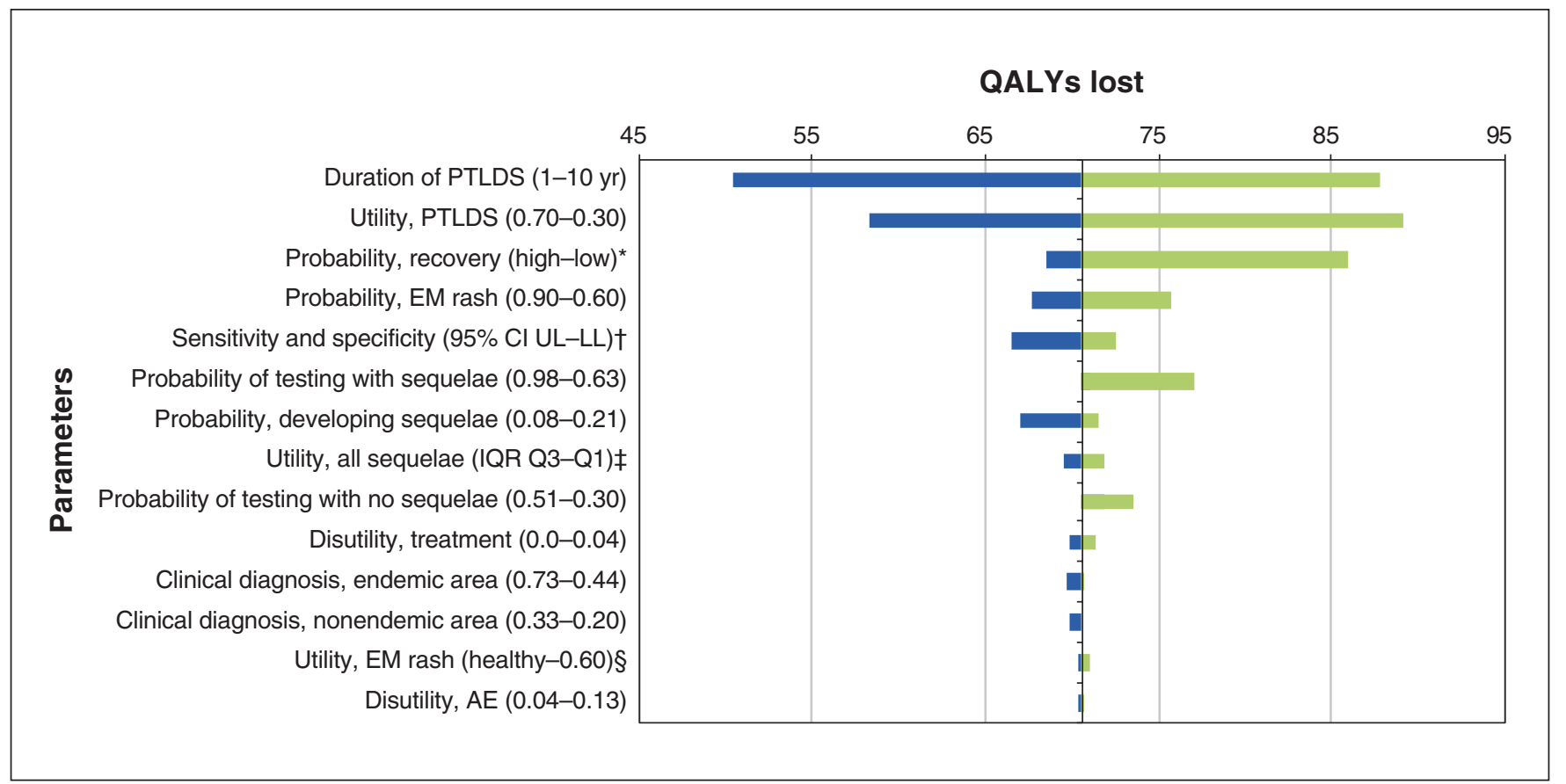

Figure 2: Sensitivity analysis of key parameters on quality-adjusted life-years (QALYs) lost over the lifetime of 100000 people in Ontario in a seeded deterministic simulation, using a base-case reference of 70.6 QALYs lost because of Lyme disease. Note: $\mathrm{AE}=\mathrm{adverse}$ event, CI = confidence interval, EM = erythema migrans, IQR = interquartile range, LL = lower limit, PTLDS = post-treatment Lyme disease syndrome, Q1 = quartile 1, Q3 = quartile 3, UL = upper limit. *Lower range is $75 \%$ of the recovery parameter (varies by sequelae). †Upper and lower limits of the $95 \% \mathrm{Cl}$ for diagnostic test characteristics (values in Table 1). $¥ Q 1$ values for low values, Q3 values for high values, simultaneously for all sequelae utility values. §Equivalent to having a utility value of a healthy person (varies by age and sex).

and pertussis (220 HALYs), a pathogen that did not contribute any years of life lost, but many year-equivalents of reduced functioning. ${ }^{34}$

Modelled scenarios also suggest that the health burden resulting from Lyme disease can be reduced by introducing interventions to lower the ecological transmission of Borrelia burgdorferi in hosts to reduce incidence rates, or by reducing the number of people who may be susceptible to Lyme disease. Previous Markov models assessing the cost-effectiveness of a vaccine for Lyme disease concluded that the disease's incidence rate greatly influences the economic value of interventions. ${ }^{9} 10$

\section{Limitations}

We may have underestimated the probability of developing sequelae as we extracted it from an Ontario cohort with laboratory-confirmed Lyme disease from 2006 to 2013, when incidence rates of Lyme disease were lower. To date, there are limited observational studies reporting health outcomes of Lyme disease in Canada; ${ }^{25,35}$ hence, we were unable to compare results of our study.

Much remains unclear about PTLDS, including its diagnosis, treatment and recovery. ${ }^{36}$ As a result, we had to assume the average duration of the PTLDS health state. In our sensitivity analysis, we showed that PTLDS (duration and utility value) influenced QALYs lost, indicating that this is an area for future research. Our model does not consider seasonality because it affects the rate, but not the severity of disease; we assumed that the health burden of Lyme disease can be simplified to result from a uniform infection risk throughout the year.

This individual-level state-transition model simulated the disease history of Lyme disease from infection to the end of life, capturing individual differences (e.g., age, sex, probability of residing in high-risk areas) to estimate population-level health burden. A modelling approach allows for the estimation of the number of infections that may not be captured by the health care system (e.g., observational studies using administrative data) capturing their expected burden and converting it to QALYs. This approach is critical in understanding the overall burden of Lyme disease as it captures the reduction in quality of life, given that Lyme disease rarely results in death. Decision-makers can adapt this model to evaluate the effectiveness, costs and value of a potential vaccine, awareness and education campaigns, improved diagnostics, or interventions to reduce the probability of an infected individual developing PTLDS.

\section{Conclusion}

Based on our model, Lyme disease infection in Ontario, Canada, contributes considerable health burden as measured by QALYs, resulting from potential sequelae, undiagnosed cases and individuals with PTLDS. The incidence rate of Lyme disease, and the duration of PTLDS and associated quality of life, were most influential to model results and should be the focus of future research and interventions. 


\section{References}

1. Why is CDC concerned about Lyme disease? Atlanta: Centers for Disease Control and Prevention; reviewed 2021 Jan. 13. Available: https://www.cdc. gov/lyme/why-is-cdc-concerned-about-lyme-disease.html (accessed 2020 Aug. 3).

2. Rochlin I, Ninivaggi DV, Benach JL. Malaria and Lyme disease: the largest vector-borne US epidemics in the last 100 years - success and failure of public health. BMC Public Health 2019;19:804.

3. Mead PS. Epidemiology of Lyme disease. Infect Dis Clin North Am 2015;29: $187-210$.

4. Lyme disease data tables: historical data. Atlanta: Centers for Disease Control and Prevention; reviewed 2021 May 5. Available: https://www.cdc.gov/lyme/ stats/tables.html (accessed 2018 Feb. 5).

5. Nelder MP, Wijayasri S, Russell CB, et al. The continued rise of Lyme disease in Ontario, Canada: 2017. Can Commun Dis Rep 2018;44:231-6.

6. Kugeler KJ, Griffith KS, Gould LH, et al. A review of death certificates listing Lyme disease as a cause of death in the United States. Clin Infect Dis 2011; 52:364-7.

7. Aucott JN. Posttreatment Lyme disease syndrome. Infect Dis Clin North Am 2015;29:309-23

8. Mac S, Bahia S, Simbulan F, et al. Long-term sequelae and health-related quality of life associated with Lyme disease: a systematic review. Clin Infect Dis 2020;71:440-52.

9. Hsia EC, Chung JB, Schwartz JS, et al. Cost-effectiveness analysis of the Lyme disease vaccine. Arthritis Rheum 2002;46:1651-60.

10. Shadick NA, Liang MH, Phillips CB, et al. The cost-effectiveness of vaccination against Lyme disease. Arch Intern Med 2001;161:554-61.

11. Meltzer MI, Dennis DT, Orloski KA. The cost effectiveness of vaccinating against Lyme disease. Emerg Infect Dis 1999;5:321-8.

12. Eckman MH, Steere AC, Kalish RA, et al. Cost effectiveness of oral as compared with intravenous antibiotic therapy for patients with early Lyme disease or Lyme arthritis. N Engl 7 Med 1997;337:357-63.

13. Guertin JR, Feeny D, Tarride JE. Age- and sex-specific Canadian utility norms, based on the 2013-2014 Canadian Community Health Survey. CMAF 2018;190:E155-61.

14. Guidelines for the economic evaluation of health technologies: Canada. 4th ed. Ottawa: Canadian Agency for Drugs and Technologies in Health (CADTH); 2017:1-76.

14. Henry B, Crabtree A, Roth D, et al. Lyme disease: knowledge, beliefs, and practices of physicians in a low-endemic area. Can Fam Physician 2012;58: e289-95.

16. For health professionals: Lyme disease. Ottawa: Public Health Agency of Canada; modified 2021 Feb. 8. Available: https://www.canada.ca/en/public -health/services/diseases/lyme-disease/health-professionals-lyme-disease.html (accessed 2021 Apr. 19).

17. Lyme disease - Serology. Toronto: Public Health Ontario; updated July 2020. Available: https://www.publichealthontario.ca/en/laboratory-services/ test-information-index/lyme-disease-serology (accessed 2020 Sept. 23).

18. Waddell LA, Greig J, Mascarenhas M, et al. The accuracy of diagnostic tests for Lyme disease in humans, a systematic review and meta-analysis of north American research. PLoS One 2016;11:e0168613.

19. Magid D, Schwartz B, Craft J, et al. Prevention of Lyme disease after tick bites. A cost-effectiveness analysis. N Engl f Med 1992;327:534-41.

20. Liu NY, Dinerman H, Levin RE, et al. Randomized trial of doxycycline vs. amoxicillin/probenecid for the treatment of Lyme arthritis: treatment of non-responders with intravenous penicillin or ceftriaxone. Arthritis Rheum 1989;32:S46

21. Steere AC, Taylor E, McHugh GL, et al. The overdiagnosis of Lyme disease. 7AMA 1993;269:1812-6.

22. Logigian EL, Steere AC. Clinical and electrophysiologic findings in chronic neuropathy of Lyme disease. Neurology 1992;42:303-11.

23. Dattwyler RJ, Volkman DJ, Halperin JJ, et al. Treatment of late Lyme borreliosis - randomised comparison of ceftriaxone and penicillin. Lancet $1988 ; 1: 1191-4$

24. Karlsson M, Hammers-Berggren S, Lindquist L, et al. Comparison of intravenous penicillin $\mathrm{G}$ and oral doxycycline for treatment of lyme neuroborreliosis. Neurology 1994:44:1203-7.

25. Shing E, Wang J, Khoo E, et al. Estimating direct healthcare costs attributable to laboratory-confirmed Lyme disease in Ontario, Canada: a populationbased matched cohort study using health administrative data. Zoonoses Public Health 2019;66:428-35.
26. Mac S, Jung J, Kopp A, et al. Estimating health burden attributable to laboratoryconfirmed Lyme disease in Ontario, Canada: a population-based matched cohort study using health administrative data. Med Decis Making 2020;40:E102-3.

27. Wormser GP, Dattwyler RJ, Shapiro ED, et al. The clinical assessment, treatment, and prevention of Lyme disease, human granulocytic anaplasmosis, and babesiosis: clinical practice guidelines by the Infectious Diseases Society of America. Clin Infect Dis 2006;43:1089-134.

28. Gold MR, Stevenson D, Fryback DG. HALYs and QALYs and DALYs, Oh My: similarities and differences in summary measures of population health. Annu Rev Public Health 2002;23:115-34.

29. Caro JJ, Briggs AH, Siebert U, et al. Modeling good research practices overview: a report of the ISPOR-SMDM Modeling Good Research Practices Task Force-1. Med Decis Making 2012;32:667-77.

30. Rebman AW, Bechtold KT, Yang T, et al. The clinical, symptom, and qualityof-life characterization of a well-defined group of patients with posttreatment Lyme disease syndrome. Front Med (Lausanne) 2017;4:224.

31. Nadelman RB, Wormser GP. Reinfection in patients with Lyme disease. Clin Infect Dis 2007;45:1032-8.

32. Jares TM, Mathiason MA, Kowalski TJ. Functional outcomes in patients with Borrelia burgdorferi reinfection. Ticks Tick Borne Dis 2014;5:58-62.

33. Johnson KO, Nelder MP, Russell C, et al. Clinical manifestations of reported Lyme disease cases in Ontario, Canada: 2005-2014. PLoS One 2018;13:e0198509.

34. Kwong JC, Ratnasingham S, Campitelli MA, et al. The impact of infection on population health: results of the Ontario burden of infectious diseases study. PLoS One 2012; 7:e44103.

35. About the network. Canadian Lyme Disease Research Network. Available: https://www.clydrn.ca/about-the-network/\# (accessed 2021 Apr. 19).

36. Rebman AW, Aucott JN. Post-treatment Lyme disease as a model for persistent symptoms in Lyme disease. Front Med (Lausanne) 2020;7:57.

Affiliations: Institute of Health Policy, Management and Evaluation (Mac, Sander), University of Toronto; THETA Collaborative (Mac, Sander), University Health Network, Toronto, Ont.; Department of Medicine (Evans), Queen's University, Kingston, Ont.; ICES Central (Evans, Sander); Public Health Ontario (Patel, Sander); Department of Laboratory Medicine and Pathobiology (Patel), University of Toronto; The Hospital for Sick Children (SickKids) (Pullenayegum); Dalla Lana School of Public Health (Pullenayegum), University of Toronto, Toronto, Ont.

Contributors: All of the authors contributed substantially to the conception of the work, data acquisition, analysis and interpretation of data. Stephen Mac drafted the work. Gerald Evans, Samir Patel, Eleanor Pullenayegum and Beate Sander revised the manuscript critically for important intellectual content. All authors gave final approval of the version to be published and agree to be accountable for all aspects of the work.

Funding: This work was supported by the Canadian Institutes of Health Research (CIHR) project grant PJT149087, held by Beate Sander. Stephen Mac is supported by a CIHR Frederick Banting and Charles Best Canada Graduate Scholarship Doctoral Award GSD-159274. Beate Sander is supported by a Canada Research Chair in Economics of Infectious Diseases (CRC-950-232429). The funders had no role in study design, data collection and analysis, decision to publish or preparation of the manuscript.

Content licence: This is an Open Access article distributed in accordance with the terms of the Creative Commons Attribution (CC BY-NC-ND 4.0) licence, which permits use, distribution and reproduction in any medium, provided that the original publication is properly cited, the use is noncommercial (i.e., research or educational use), and no modifications or adaptations are made. See: https://creativecommons.org/licenses/ by-nc-nd/4.0/

Data sharing: All data generated or analyzed during this study are included in this article.

Supplemental information: For reviewer comments and the original submission of this manuscript, please see www.cmajopen.ca/content/9/4/ E1005/suppl/DC1 